\title{
FLUJOS DE AGUA, FLUJOS DE CAPITAL: SISTEMAS DE ABASTECIMIENTO Y GOBERNANZA DEL AGUA EN MADRID Y BARCELONA
}

\author{
Hug March y David Saurí \\ Departament de Geografía, Universitat Autònoma de Barcelona
}

\section{RESUMEN}

El agua es un elemento clave para el sustento del metabolismo de las ciudades. Sin embargo, los orígenes, métodos de «producción», y modelos de gestión del flujo de urbano de agua pueden variar geográficamente. En este artículo se comparan los sistemas de abastecimiento y gestión de agua potable en las dos principales regiones metropolitanas del Estado: Barcelona y Madrid. En Barcelona, el agua procede de distintas fuentes entre las cuales destaca la desalación, vigente desde el verano de 2009. En Madrid, el sistema de abastecimiento depende fuertemente de las aguas superficiales procedentes de diversos ríos que atraviesan la Comunidad. Por lo que respeta a la gestión de estos sistemas el presente trabajo hace hincapié sobre las importantes diferencias que giran entorno al distinto balance entre participación pública y privada en el ciclo del agua en ambas áreas. Finalmente el artículo reflexiona sobre los puntos de convergencia y divergencia entre ambos modelos de abastecimiento así como sus perspectivas de futuro.

Palabras clave: Agua, urbanismo, historia, gobernanza, Barcelona, Madrid.

\begin{abstract}
Water is a key element to sustain the metabolism of cities. However, the sources, the methods of water «production» as well as the management models of the urban water flow could differ widely. In this paper we compare the urban water supply systems of the two most important metropolitan regions of Spain: Barcelona and Madrid. In Barcelona, water comes from different sources, with an important role of desalination since the summer of 2009. In Madrid, water supply depends heavily on the surface waters of different rivers flowing through the region. In what concerns the management of these systems, the paper points out the important differences on the governance of the water cycle, and the relationships between public and private interests. To conclude, the article reflects on the convergence and divergence between both water governance models and attempts to offer some insights regarding future prospects.
\end{abstract}

Key words: Water, urbanism, history, governance, Barcelona, Madrid. 


\section{Introducción}

El proceso de urbanización no puede entenderse sin incorporar al análisis el proceso paralelo de movilización de recursos hídricos para alimentar el metabolismo de las ciudades. Este metabolismo, sin embargo, dependerá estrechamente de las características de la urbanización y, en particular de la forma urbana (compacta o dispersa) adoptada en cada fase del proceso. Desarrollos urbanos de tipo compacto favorecerán probablemente consumos de agua por cápita menores y, por tanto, requerirán infraestructuras hidráulicas menos onerosas desde un punto de vista económico, social y ambiental. En cambio, el crecimiento urbano difuso tenderá a generar mayores consumos por cápita principalmente por la existencia de usos exteriores del agua como los derivados de jardines y piscinas. De ahí, por ejemplo, las enormes diferencias existentes entre el consumo de agua en las áreas urbanas de ciertos países como los EEUU (hasta 1.000 litros por persona y día en ciudades de Arizona o California) y el consumo de agua de ciudades europeas de modelo compacto.

Este esquema general debe matizarse sin embargo en función de las trayectorias específicas de la urbanización en distintos ámbitos urbanos. El presente artículo compara los casos de Barcelona y Madrid, tanto por lo que se refiere a las tendencias recientes en el consumo de agua como a las alternativas planteadas para asegurar este consumo en el futuro. Ambas ciudades han conocido cambios importantes en su crecimiento urbano reciente y han optado también por distintas soluciones para asegurar los abastecimientos de agua. Igualmente, en ambas ciudades se configuran distintos sistemas de gobernanza del agua (en Madrid, el abastecimiento se encuentra en manos de una empresa pública mientras que en Barcelona se halla en manos privadas) que muestran sin embargo, ciertos procesos de convergencia. En definitiva, las dos mayores ciudades españolas constituyen un ejemplo muy interesante de las relaciones históricas entre proceso de urbanización y desarrollo de recursos hídricos, así como de convergencia entre dos sistemas de gobernanza del agua a priori muy diferentes.

El artículo se halla organizado de la siguiente forma. En primer lugar, presentamos los rasgos principales de la historia de las relaciones entre urbanización y agua en ambas ciudades. En segundo lugar, examinamos las alternativas de abastecimiento de agua en Barcelona y Madrid, todas ellas basadas en el aumento de la oferta sea a través de medios convencionales (como la regulación de las aguas superficiales en Madrid), o bien a través de medios alternativos (como la desalación en Barcelona). En tercer lugar, nos centraremos en examinar las similitudes y diferencias en lo que se refiere a la gobernanza de los recursos hídricos en cada ciudad, destacando la singularidad de una compañía privada bajo un regulador público de perfil izquierdista (Barcelona) y de una compañía pública regulada por un gobierno regional con muchas simpatías hacia la privatización de servicios públicos (Madrid). Finalmente, en las conclusiones intentaremos contraponer las experiencia de ambas ciudades en la gestión del agua y el futuro que parece abrirse y que indicaría una cierta aproximación entre ambos sistemas.

\subsection{Barcelona: suburbanización y discurso de sequía permanente}

En las últimas décadas, la mayor parte del crecimiento urbano europeo y en especial de la mitad meridional del continente se ha producido bajo la modalidad de baja densidad (EEA 2006). En Barcelona como en Madrid, este cambio de modelo urbano, y no sólo el aumento de la población, han jugado un papel clave en determinar las necesidades hídricas de la ciudad. Horacio Capel (2007), argumenta que, en el caso de Barcelona, los antecedentes del modelo en baja densidad que caracteriza la periferia metropolitana se encuentran en la 
denominada ciudad-jardín, de gran popularidad entre ciertos urbanistas durante la década de 1930. Sin embargo, la forma urbana que acogió a los grandes contingentes inmigratorios de las décadas de 1950, 1960 y 1970 no fue la de la «caseta i hortet» presagiada por el presidente Macià durante los años de la República, sino los enormes bloques de viviendas de superficie exigua levantados en los barrios y municipios periféricos de Barcelona. La densidad extrema alcanzada por Barcelona y algunos de sus municipios colindantes como L'Hospitalet de Llobregat o Santa Coloma de Gramenet, y una cierta mejora en el nivel de vida durante las décadas de 1960 y 1970 (concretada sobretodo en la posibilidad de adquirir un automóvil) propició un proceso de invasión de las áreas rurales y montañosas más cercanas al área metropolitana y la aparición de las denominadas urbanizaciones o viviendas secundarias construidas para escapar de la sobredensificación de las barriadas populares. La misma falta de planificación y control y los intereses claramente especulativos de la iniciativa privada, que habían facilitado el crecimiento vertical en la ciudad densa, fue responsable del desarrollo de estos nuevos espacios urbanos que, en mayor o menor medida, si se acomodaban más a la filosofía de la ciudad jardín, aunque fuera de manera precaria (aún hoy muchas urbanizaciones carecen de servicios básicos como el alcantarillado) y limitada a los fines de semana y vacaciones. De este modo, la génesis de la suburbanización y dispersión urbanas en la Región Metropolitana de Barcelona tiene su origen más inminente en estas décadas de 1960 y 1970, aunque existen unas primeras manifestaciones a inicios del siglo XX (Barba y Mercadé 2006). Con el tiempo, estas residencias secundarias se convirtieron en viviendas principales y contribuyeron a la consolidación del modelo urbano de baja densidad en la periferia de la aglomeración barcelonesa.

Esta primera etapa del proceso de suburbanización en Barcelona se cierra con las crisis económicas y políticas de la década de 1970 que ralentizaron el crecimiento y también el crecimiento urbano. A partir de la segunda mitad de la década de 1980, la Región Metropolitana de Barcelona recupera el pulso económico y empieza a experimentar un proceso de redistribución de la población y de las actividades económicas, con la subsiguiente pérdida de población en los centros urbanos más densos y el crecimiento de la población en los núcleos más periféricos (ver tabla 1$)$.

Tabla 1

EVOLUCIÓN POBLACIÓN REGIÓN METROPOLITANA DE BARCELONA (RMB) POR CORONAS, 1975-2008

\begin{tabular}{|c|c|c|c|c|c|c|c|c|}
\hline & 1975 & 1981 & 1986 & 1991 & 1996 & 2001 & 2005 & 2008 \\
\hline Barcelona & 1.751 .136 & 1.752 .627 & 1.701 .812 & 1.643 .542 & 1.508 .805 & 1.505 .325 & 1.593 .075 & 1.615 .908 \\
\hline $\begin{array}{l}1^{\text {era }} \text { corona } \\
(\mathrm{AMB})^{1}\end{array}$ & 3.008 .770 & 3.145 .013 & 3.083 .353 & 3.037 .763 & 2.904 .941 & 2.862 .708 & 3.135 .758 & 3.186 .461 \\
\hline $2^{\mathrm{a}}$ corona & 1.010 .943 & 1.093 .863 & 1.146 .174 & 1.226 .659 & 1.323 .107 & 1.525 .016 & 1.634 .422 & 1.742 .391 \\
\hline Total RMB & 4.019 .713 & 4.238 .876 & 4.224 .527 & 4.264 .422 & 4.228 .048 & 4.387 .724 & 4.770 .180 & 4.928 .852 \\
\hline
\end{tabular}

Fuente: Elaboración propia a partir de Institut d'Estadística de Catalunya.

1 Incluye la ciudad de Barcelona. 
Entre 1981 y 2001 la segunda corona metropolitana de Barcelona creció de manera importante (Algaba, 2001), con un flujo neto de población equivalente a la pérdida de población del Ârea Metropolitana de Barcelona (Sánchez, 2003). Esta tendencia, vigente desde la década de 1980, parece que se vuelve a reequilibrar con la llegada de importantes contingentes de inmigrantes a la áreas centrales, especialmente entre 2001 y 2007 (March et al., en prensa). Así, los procesos de suburbanización convergen en el tiempo con una reurbanización de las áreas centrales (Arias y Borja, 2005), de modo que, durante los últimos años, el crecimiento demográfico es perceptible tanto en los núcleos densos que habían perdido población durante las décadas de 1980 y 1990 como en los nuevos espacios periféricos. Paralelamente se producen también importantes cambios sociodemográficos. El núcleo denso de la RMB tiende a quedar en manos de población autóctona envejecida y de inmigrantes de escasos recursos, mientras que las periferias han constituido el destino de las capas de población jóvenes y relativamente acomodadas que, por diversos motivos, han abandonado los núcleos centrales.

Estos cambios sociodemográficos y territoriales han comportado a su vez cambios importantes en el consumo de agua (March y Saurí, 2009, 2010; March et al., en prensa). Así, la creciente heterogeneidad del modelo urbano configura una geografía muy desigual del consumo doméstico de agua en la RMB, con dispendios que escasamente superan los 100 litros por cápita y día (lpd) en ciudades compactas hasta los más de 300 lpd (en 2006²) de núcleos residenciales de baja densidad como Matadepera o Sant Andreu de Llavaneres. Estos casos extremos del espectro representan modelos urbanos (y sociales) que se encuentran más cerca de Los Angeles o Perth que de cualquier ciudad mediterránea. La presencia masiva de jardines atlánticos así como de piscinas explica en gran medida estas diferencias (Domene et al. 2005, Domene y Saurí 2006, Parés-Franzi et al., 2006, Domene y Saurí 2007).

A este contex to demográfico, social y territorial caracterizado por una tendencia al aumento en el consumo de agua se añade un entorno climático que, en las últimas décadas, ha resultado poco propicio a las precipitaciones y un sistema de suministro cuya capacidad apenas ha cambiando durante los últimos veinte años. Todos estos factores han repercutido en la multiplicación de los episodios de sequía que la Región Metropolitana de Barcelona ha sufrido durante las décadas de 1990 y 2000 hasta el punto que en 2008 se tuvo que recurrir al abastecimiento por medio de buques cisterna. En los últimos años el Gobierno catalán ha debido restringir temporalmente ciertos usos, incluso urbanos y domésticos, como el riego de jardines públicos y privados.

Como argumenta Kallis (2008), la sequía no es solamente un fenómeno físico, sino que también tiene un fuerte componente humano y social. En este sentido, argumentamos que la suburbanización está incrementando de manera notable el metabolismo hídrico de Barcelona y alterando el ya por si frágil equilibrio hídrico de la región. Así, podemos afirmar que la escasez de agua en la MRB no sólo es física sino que parcialmente está socialmente construida y producida por tendencias sociodemográficas, económicas y territoriales como las que se han comentado anteriormente.

\subsection{Madrid: una gran región metropolitana a la que dar de beber}

La historia del suministro de agua a la capital, española también se encuentra íntimamente ligada al proceso de urbanización de lo que actualmente es una de las regiones urbanas más importantes de Europa: la Comunidad Autónoma de Madrid (CAM) y sus más de cinco millones de habitantes. En una tendencia parecida a la de Barcelona, desde

\footnotetext{
2 Datos de la Agència Catalana de l'Aigua.
} 
1985 hasta la actualidad ha existido un gran crecimiento en las áreas periféricas de Madrid, reflejando, en parte, un proceso de reconversión de vivienda secundaria a vivienda principal. El reverso de este proceso ha sido la pérdida de población en términos relativos y absolutos de la ciudad de Madrid (ver figura 1). En 2005 la Agencia Europea de Medio Ambiente califico la región de Madrid como uno de los «hotspots» de desarrollo urbano debido a su extraordinario crecimiento en la década de 1990 (EEA, 2005), acompañado de una importante reducción de la tierras agrícolas (Plata Rocha et al., 2009). Así, hoy en día podemos decir que la CAM es una región que sigue patrones de «urban sprawl» (García Palomares y Gutiérrez Puebla 2007), en parte atribuido a las opciones de planeamiento territorial (López de Lucio 2003, European Environment Agency (EEA) 2006). El informe Urban Sprawl in Europe: the ignored challenge [Urban Sprawl en Europa: el desafío ignorado] de la Agencia Europea de Medio Ambiente argumenta que este proceso «está modificando profundamente Madrid de una manera insostenible», añadiendo que «el desarrollo sostenible de la región de Madrid sólo puede ser conseguido con un escenario de desarrollo compacto» (EEA, 2006:24).

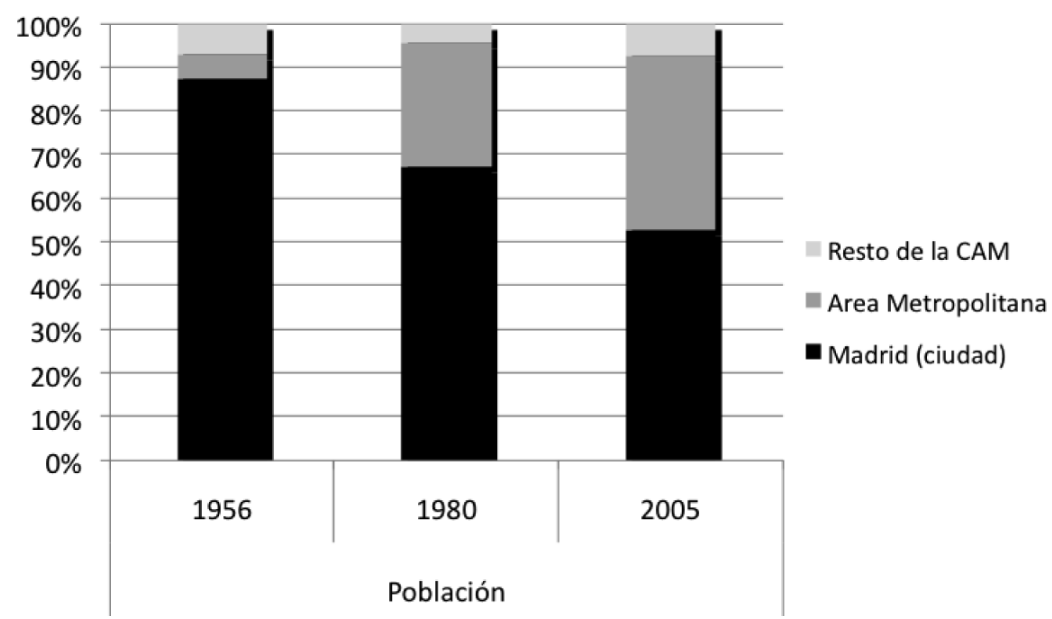

Figura 1. Distribución de la población (en términos relativos) en la CAM, 1956-2005. Fuente: Elaboración propia a partir de Naredo y García Zaldívar (2008).

El metabolismo hídrico de la región de Madrid ha seguido el paso de este incremento poblacional y este proceso de suburbanización. En la historia reciente del suministro de agua en la CAM detectamos una fecha clave, que marca un punto de inflexión en los patrones de consumo: la sequía de 1992 (figura 2). Si bien los factores que han contribuido a este importante descenso del consumo y mantenimiento de una menor demanda no están claramente identificados, se han destacado los efectos de las campañas de educación ambiental, la mayor eficiencia de las industrias, o el efecto permanente de la sequía en los ciudadanos (muchos de éstos habrían normalizado sus hábitos de consumo durante estos períodos). Si nos atendemos a los estudios que se han llevado a cabo en Barcelona (March y Saurí, 2010, March et al., en prensa) podemos aventurar también que parte de este decrecimiento en el consumo de agua se ha debido a cambios demográficos y en particular a la mayor concentración de población envejecida e inmigrante en los espacios centrales de la metrópolis. 


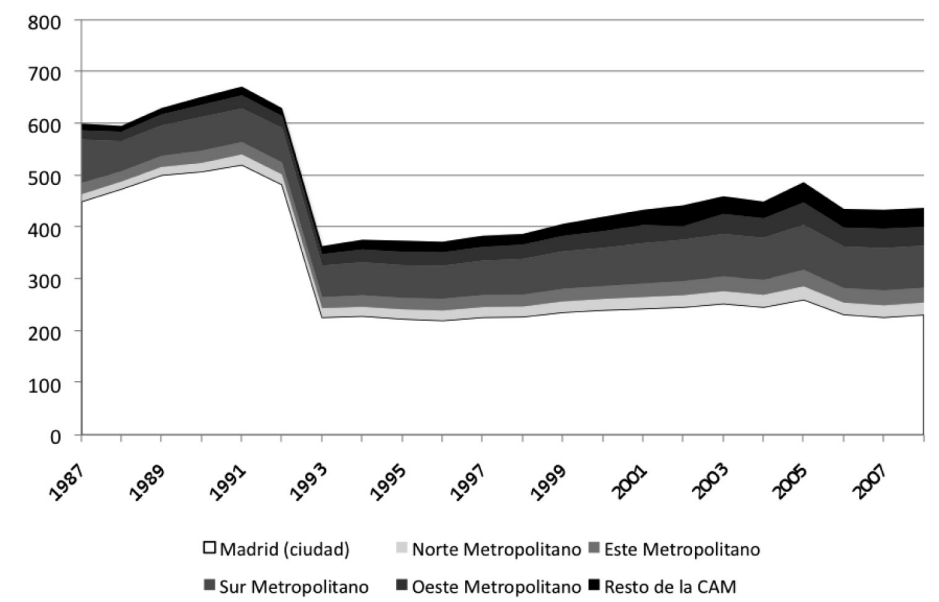

Figura 2. Evolución de la demanda de agua en la CAM por zonas, en hm³ , 1987-2008. Fuente: Elaboración propia a partir de Instituto de Estadística de Madrid y Canal de Isabel II.

Sin embargo el decrecimiento generalizado del consumo de agua en la región de Madrid nos lleva a plantearnos la cuestión de si realmente estamos frente a un menor uso de agua o solamente se trata de un desplazamiento geográfico de los puntos de consumo. El concepto de agua virtual (Allan, 1998), entendida como el agua que se necesita durante el proceso de producción de un bien o servicio, es utilizado por Naredo et al., (2008) para mostrar que Madrid ha ido consumiendo progresivamente más agua de este tipo y por el contrario utilizando menos recursos locales/regionales. Así, mientras el consumo anual por cápita de agua extraída en la CAM ha decrecido de 261 a $191 \mathrm{~m}^{3}$, el consumo de agua virtual ha incrementado de forma muy notable, incrementando la huella hídrica (Chapagain et al. 2006, Hoekstra and Chapagain 2007) de Madrid de $1.169 \mathrm{~m}^{3}$ en 1984 a $1.667 \mathrm{~m}^{3}$ por capita y año en 2005 (Naredo et al., 2008). En otras palabras, mientras el consumo de agua de red de un madrileño en 2005 era de unos 523 lpd, la huella hídrica de cada ciudadano de la CAM supera los 4.500 lpd (Naredo et al. 2008).

De la misma manera, también es importante interrogarse, igual que en el caso de Barcelona donde se consume el agua. El sector doméstico representa, con mucho, el consumidor más importante de los recursos hídricos de la CAM (figura 3). Sin embargo, este consumo no es homogéneo sino que difiere a lo largo de la región de Madrid.

En 2004 y de acuerdo con el Instituto Nacional de Estadística, la CAM contaba con más de 350.000 casas apareadas o chalets. La mayor parte de estas viviendas, unas 200.000 son utilizadas como residencias principales (Diario ABC, 27 de Mayo de 2006). De acuerdo con el «Manual de Abastecimiento del Canal de Isabel II» (Cubillo González e Ibáñez Carranza 2005[2003]) la viviendas unifamiliares en la CAM presentan consumos de agua por cápita notablemente superiores al consumo de las viviendas plurifamiliares: mientras la dotación por cápita de un piso en la CAM se sitúa alrededor de los 400 litros por día, ésta se ve aumentada en 200 litros en una vivienda unifamiliar (idem). Aunque las viviendas unifamiliares sólo representen un $20 \%$ del consumo doméstico (datos de 2004, idem), su efecto en el metabolismo hídrico de la región es importante y puede aún volverse más importante si el modelo en baja densidad prospera en la región, tal y como contemplan algunos escenarios planteados por la Agencia Europea de Medio Ambiente 
(European Environment Agency (EEA) 2006) y se propugna también desde los entornos políticos con responsabilidades de gobierno (ver, por ejemplo, Diario ABC, Martes 8 de Mayo 2007, p. 10, «Aguirre anuncia un nuevo urbanismo que pondrá fin a las viviendas altas por ley»; también Elmundo.es, Lunes 5 de Mayo de 2007, «Aguirre limitará a cuatro plantas los nuevos edificios residenciales).

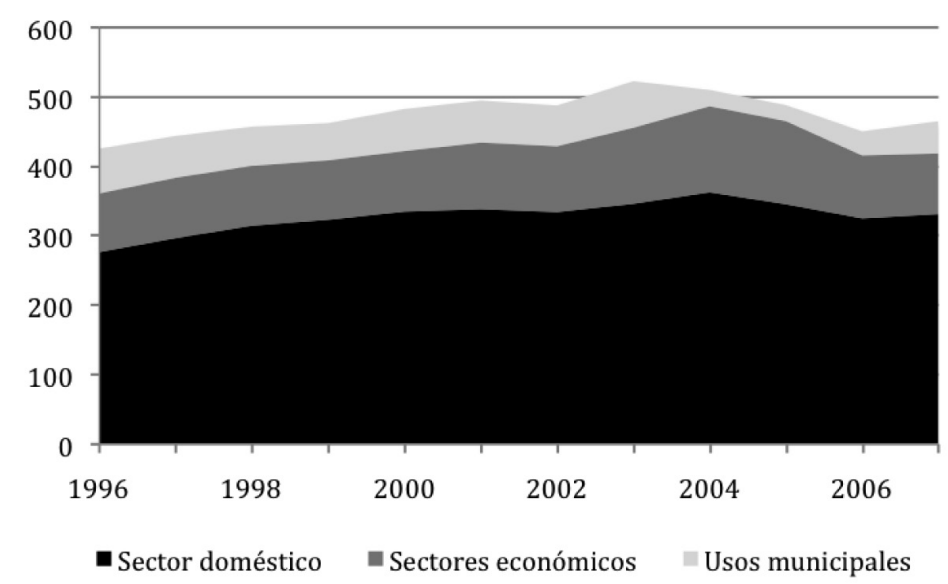

Figura 3. Distribución de la demanda de agua por sectores, en hm³ , 1996-2007. Fuente: Elaboración propia a partir de Instituto de Estadística de Madrid e Instituto Nacional de Estadística.

\section{Las infraestructuras para la obtención de agua}

Por urbanización del suministro de agua entendemos el proceso de movilización de recursos hídricos para mantener el ritmo de crecimiento urbano. Matthew Gandy argumenta que la arqueología del flujo de agua urbano sirve para «iluminar el funcionamiento de las sociedades modernas en toda su complejidad» (2002:22) y que: «la historia de las ciudades puede ser leída como la historia del agua» (Gandy, 2002:22). La historia de los abastecimientos hídricos de Madrid y Barcelona refleja perfectamente estas aseveraciones puesto que cada etapa del proceso de urbanización ha ido acompañada de cambios en los sistemas de abastecimiento y la incorporación de nuevas fuentes de suministro de agua. Ambas ciudades han respondido a las presiones de la urbanización mediante políticas orientadas a incrementar el aumento de los recursos disponibles y sólo muy recientemente, las políticas de contención de la demanda han empezado a despertar cierto interés. Con todo, Madrid y Barcelona presentan diferencias importantes en relación a las fuentes de suministro. Así, mientras Madrid ha recurrido históricamente a las aguas superficiales, en Barcelona el peso de las aguas subterráneas y recientemente de otros recursos como la desalación ha sido más notable.

\subsection{El Viejo estructuralismo hidráulico: embalses y trasvases}

La regulación de las aguas superficiales ha constituido el eje vertebrador del suministro de agua a Barcelona y Madrid, más pronunciado en el segundo caso que en el 
primero. Por lo que se refiere a Barcelona, históricamente la ciudad dependió de pequeños acueductos procedentes de cuencas cercanas (como el denominado Rec Comtal) y, sobretodo de las aguas subterráneas de su entorno inmediato, como los pozos del valle del Llobregat. A principios de la década de 1950 y para atender una demanda cada vez más elevada, la Sociedad General de Aguas de Barcelona obtuvo la primera concesión para detraer agua directamente del río Llobregat. El Plan de Aguas de Cataluña de 1957 diseñado por el ingeniero Victoriano Muñoz Oms bajo la influencia del recién nombrado alcalde de Barcelona José María de Porcioles, se propuso aumentar la regulación de Llobregat mediante la construcción de nuevos embalses y, muy especialmente, establecer la conexión de la cuenca del río Ter con la aglomeración barcelonesa mediante un acueducto de más de $100 \mathrm{~km}$ de longitud. Finalmente inaugurado en 1966, el denominado «Trasvase del Ter» vino a paliar las necesidades de una urbe que entonces crecía a ritmos asiáticos. A finales de la década de 1960 y bajo unas previsiones que auguraban problemas serios de abastecimiento de agua para 1985, se empezó a estudiar la opción de un trasvase de mayor envergadura, esta vez con caudales procedentes del río Ebro. En 1973 se presentó un primer anteproyecto de trasvase de más de 1.000 hm³ anuales al área de Barcelona que fue recibido con rechazo en Aragón y en la parte catalana del Ebro. La crisis económica de la segunda mitad de la década y los cambios políticos motivaron la cancelación del proyecto.

Tabla 2

EVOLUCIÓN HISTÓRICA DE LAS PRESAS POR CUENCA DE LA REGIÓN METROPOLITANA DE BARCELONA

\begin{tabular}{|llrr|}
\hline Cuenca & Presa & En operación desde & Capacidad $\left(\mathbf{e n ~}^{\mathbf{3}} \mathbf{\text { }}\right)$ \\
\hline \multirow{4}{*}{ Ter } & Sau & 1962 & 151,3 \\
& Susqueda & 1968 & 233 \\
& Pasteral, el & 1962 & 2 \\
& Total Ter & & $\mathbf{3 8 6 , 3}$ \\
\hline \multirow{3}{*}{ Llobregat } & Baells, la & 1976 & 109,5 \\
& Llosa del Cavall, la & 1997 & 79,4 \\
& Sant Ponç & & 24,4 \\
& Total Llobregat & & $\mathbf{2 1 3 , 3}$ \\
\hline TOTAL & & & $\mathbf{5 9 9 , 6}$ \\
\hline
\end{tabular}

Fuente: Elaboración propia a partir de Agència Catalana de l'Aigua.

A falta de un segundo trasvase, el suministro de agua para Barcelona pasó a centrarse en aumentar la regulación del Llobregat mediante el pantano de La Baells (inaugurado en 1976) y los pantanos de Sant Ponç y La Llosa de Cavall, en el Cardener (tabla 2). Desde la década de 1990 no se ha producido ningún aumento substancial de la oferta de aguas superficiales para Barcelona lo cual no quiere decir que no hayan abundado los proyectos para tal finalidad. Descartada una mayor regulación de unas cuencas internas mediterráneas exhaustas, los trasvases se erigen como la solución preferida por administraciones y la élite económica del país. A mediados de la década de 1990 se empieza 
a debatir un proyecto de trasvase que abandona la opción del Ebro o de sus afluentes catalanes para dirigir la atención hacia el Ródano, en Francia. La opción del Ródano, impulsada con entusiasmo por el gobierno de Convergència i Unió, disfrutaba según sus proponentes de numerosas ventajas frente a la opción de los ríos pirenaicos o del Ebro. En primer lugar, los problemas de garantía acerca de los caudales a trasvasar se evitaban en este caso por el tamaño y regularidad del Ródano. En segundo lugar, la oposición social a este trasvase se veía reducida a grupos de los dos extremos del espectro político, al asegurarse a los agricultores del mediodía francés que en ningún caso el agua se destinaría al regadío. Sin embargo, la defensa del Ródano tenía otras razones, esta vez de orden geopolítico. Desde hacía algunos años, el gobierno catalán había aumentado la colaboración con la región francesa de Languedoc-Rousillon en el marco de la estrategia regional europea. El trasvase del Ródano venía a significar un paso importante en estrechar la política regional puesto que por primera vez se alcanzaba un acuerdo sobre un recursos estratégico a nivel regional y no nacional. Así, el Ródano podría contemplarse como un ejemplo de colaboración entre la Europa de las regiones, un nivel emergente y preferible según muchos a la Europa de los estados. Con todo, la parte francesa podía tener motivaciones de otro orden, sobre todo económicas. Durante la década de 1980 y a raíz del gran crecimiento poblacional del sureste francés, se pensó en el Ródano para el suministro de agua a las ciudades meridionales de Montpellier, Narbona y Perpiñán. A tal efecto se creó la Compagnie du Bas Rhône Languedoc (BRL). El agua del Ródano llegó a Montpellier pero no a las ciudades más meridionales, en parte por crecientes problemas financieros de la empresa BRL. El proyecto de transvasar agua a Barcelona aseguraría la llegada de agua a las capitales meridionales y solventaría estos problemas. Sin embargo, el proyecto necesitaba como condición sine qua non un acuerdo entre los estados español y francés, acuerdo que chocó contra la negativa del gobierno del Partido Popular. Por otra parte, una comisión de expertos creada por el gobierno de la Generalitat catalana para evaluar el proyecto de trasvase señaló el gran coste económico de un acueducto de más de $300 \mathrm{~km}$ de longitud y que las necesidades de agua de Barcelona podían quedar cubiertas con otras opciones. La prioridad otorgada a la desalación en detrimento de los trasvases mostrada por el PSOE tras las elecciones de 2004 acabó por postergar el proyecto del Ródano del cual, sin embargo, todavía no se ha dicho la última palabra.

La otra gran alternativa para solucionar los déficits hídricos de la Región Metropolitana de Barcelona era recuperar el trasvase del Ebro aunque con unas dimensiones mucho más reducidas que las del proyecto de 1973. El trasvase del Ebro fue la piedra angular del Plan Hidrológico Nacional aprobado bajo el gobierno del Partido Popular en 2001 y, como es sabido, dio lugar a uno de los enfrentamientos sociales y territoriales más enconados de la historia española reciente. Aquí no es el lugar para detenerse en los pormenores de este proyecto (ver, por ejemplo, Gil Olcina y Rico Amorós, 2008 y Arrojo 2003). Sin embargo, si resulta preciso insistir en que la derogación del trasvase del Ebro tras la llegada al poder del PSOE en 2004 y su sustitución por la desalación en modo alguno ha cerrado el debate sobre el agua en España tal y como se ha visto recientemente con la patrimonialización del agua por parte de varias Comunidades Autónomas en la revisión de sus estatutos de autonomía.

Sea como fuere, lo cierto es que Barcelona no tuvo acceso al agua del Ebro o del Ródano y tampoco ocupó un lugar prioritario en la construcción de las nuevas desaladoras del Programa AGUA cuyo destino preferente fueron las áreas levantinas para compensar así la cancelación del trasvase del Ebro. Nuevos episodios de indigencia pluviométrica durante la década de 2000 corroboraron la precariedad de los abastecimientos de agua a Barcelona que, mientras tanto, había visto aumentada su población en más de un millón de 
habitantes. La sequía de 2007-2008, que puso la ciudad al borde de los cortes domiciliarios, mostró los límites del sistema y halló en la desalación y no los trasvases (los proyectos para abastecer a Barcelona durante el periodo más crítico del episodio de sequía fueron de nuevo y virulentamente contestados desde la cuenca del Ebro) la alternativa para evitar crisis hídricas futuras.

Madrid también ha optado por la regulación de las aguas superficiales como principal instrumento para el suministro de agua a la ciudad. Mientras Barcelona no recibió aguas superficiales hasta la mitad del siglo XX (con excepción del Rec Comtal medieval), Madrid basó la fundación de su suministro moderno de agua en las aguas del río Lozoya, justo un siglo antes. Con este proyecto se creó lo que es hoy el mayor suministrador público de agua del Estado: el Canal de Isabel II. El crecimiento de la ciudad, a través del Ensanche de Castro (contemporáneo al Ensanche de Cerdà en Barcelona) y de las anexiones territoriales, así como los frecuentes periodos de sequía, impulsó a regular de manera integral el río Lozoya durante el siglo XX. A finales de los años 60 del siglo XX, el sistema hídrico de Madrid contaba con cuatro presas en el Lozoya (Riosequillo, El Pontón, El Villar y Puentes Viejas), una en el Jarama (el Vado), una en el Guadalix (el Vellón) y una en el Manzanares (Santillana). También se incorporó puntualmente agua de otros ríos, como el Alberche. En la década de 1970 se materializaron el trasvase del Guadarrama hacía el Aulencia y la construcción de la presa de Valmayor. Paralelamente también se inició la construcción de la mayor presa (para uso urbano) de la región, el Atazar en el Lozoya con una capacidad alrededor de 426 hm³ (Martínez Vázquez de Parga, 2001). A partir de 1980 con la creación de la Comunidad Autónoma de Madrid y la reconfiguración del Canal de Isabel II (Real Decreto 1873/84) se puede observar un cambio de paradigma que tiene en cuenta el ciclo integral del agua así como la preservación del medio ambiente y el énfasis en el saneamiento y la reutilización.

A finales de la década de 1980 el sistema de abastecimiento del Canal de Isabel II, que suministraba a la mayor parte de la Comunidad, tenía una capacidad de almacenamiento de unos $1.000 \mathrm{hm}^{3}$, con dotaciones por persona alrededor de los $320 \mathrm{lpd}$. En 1992-93 una fuerte sequía volvió a encender todas las alarmas y se recurrió a la construcción de nuevos pozos (Martínez Vázquez de Parga, 2001). Recordemos que desde la construcción de la presa de Valmayor en 1976, en la Comunidad de Madrid no se había realizado otra gran obra de abastecimiento, sólo dos pequeñas presas, con una capacidad alrededor de $15 \mathrm{hm}^{3}$ : la Aceña (en la provincia de Ávila) y los Morales (ABC, Domingo 21 de Marzo de 1993, p. 78 ; ver tabla 3 ). En este sentido, se reabrieron antiguos proyectos más allá de las fronteras regionales, como la regulación de la parte alta del Jarama, con la presa de Matallana (ya en Guadalajara), y la parte alta del Sorbe. Sin embargo tensiones entre la administración central y la regional, así como informes negativos de impacto ambiental, bloquearon el proyecto del Matallana.

Por tanto, el crecimiento de la metrópolis se ha visto acompañado por sucesivas crisis hídricas que se han saldado acudiendo incluso a la construcción de embalses en la red fluvial vecina. Paradójicamente, aunque la cuenca del Lozoya es la más regulada de la región (figura 4), suministra a la CAM una cantidad similar de agua que la cuenca del Jarama (figura 5).

Con una garantía de abastecimiento bastante superior al caso de Barcelona, Madrid mira ahora más allá de la CAM, hacia el Alberche, Sorbe, Guadarrama y Jarama, pero sobre todo al Tajo (Nota de prensa Canal de Isabel II, 27 de Abril 2009, «El Canal de Isabel II tomará agua del Tajo por primera vez en su historia») como reservorio futuro de agua para las necesidades de la capital, cifradas alrededor de $450 \mathrm{hm}^{3}$ por el gobierno autonómico para 2016 (ABC, Viernes 25 de Noviembre de 2005, p. 38). 
Tabla 3

EVOLUCIÓN HISTÓRICA DE LAS PRESAS POR CUENCA QUE ABASTECEN LA CAM

\begin{tabular}{|llrr|}
\hline Cuenca & Presa & En operación desde & Capacidad $\left(\mathbf{e n ~}^{\mathbf{3}} \mathbf{~}^{\mathbf{3}}\right)$ \\
\hline \multirow{3}{*}{ Lozoya } & El Vilar & 1879 & 22,4 \\
& Puentes Viejas & 1939 & 53 \\
& Riosequillo & 1958 & 50 \\
& Pinilla & 1967 & 38,1 \\
Jarama & El Atazar & 1972 & 425 \\
Guadalix & El Vado & 1960 & 55,7 \\
Manzanares & Pedrezuela & 1968 & 40,9 \\
& Manzanares el Real & 1912 y & 91,2 \\
Guadarrama & Navacerrada & 1969 & 11 \\
& Navalmedio & 1969 & 0,7 \\
& La Jarosa & 1969 & 7,2 \\
Alberche & Valmayor & 1976 & 134,4 \\
& Los Morales & 1988 & 2,3 \\
TOTAL & La Aceña & 1991 & 23,7 \\
\hline
\end{tabular}

Fuente: Elaboración propia a partir de datos del Canal de Isabel II.

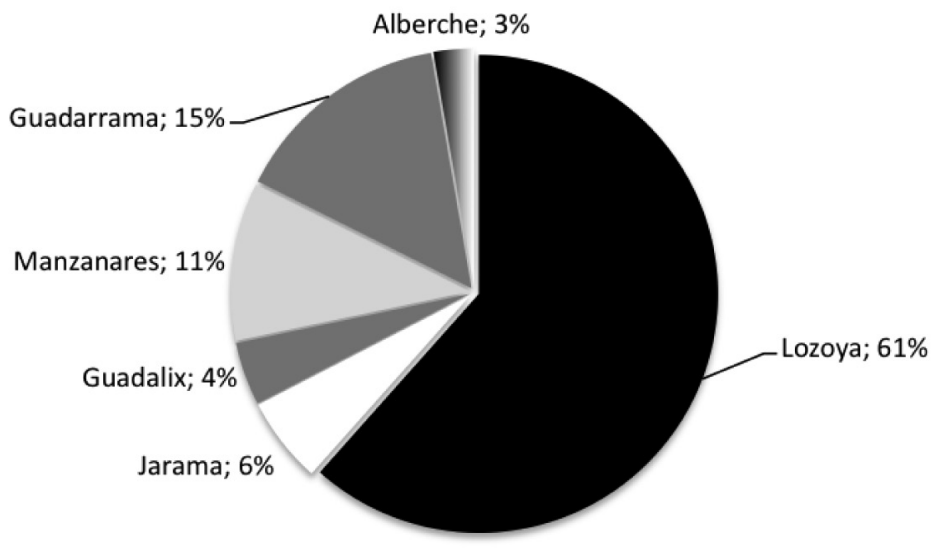

Figura 4. Capacidad de almacenamiento en cada cuenca en relación a la capacidad total de la CAM, en porcentaje, 2009.

Fuente: Elaboración propia a partir de datos del Canal de Isabel II. 


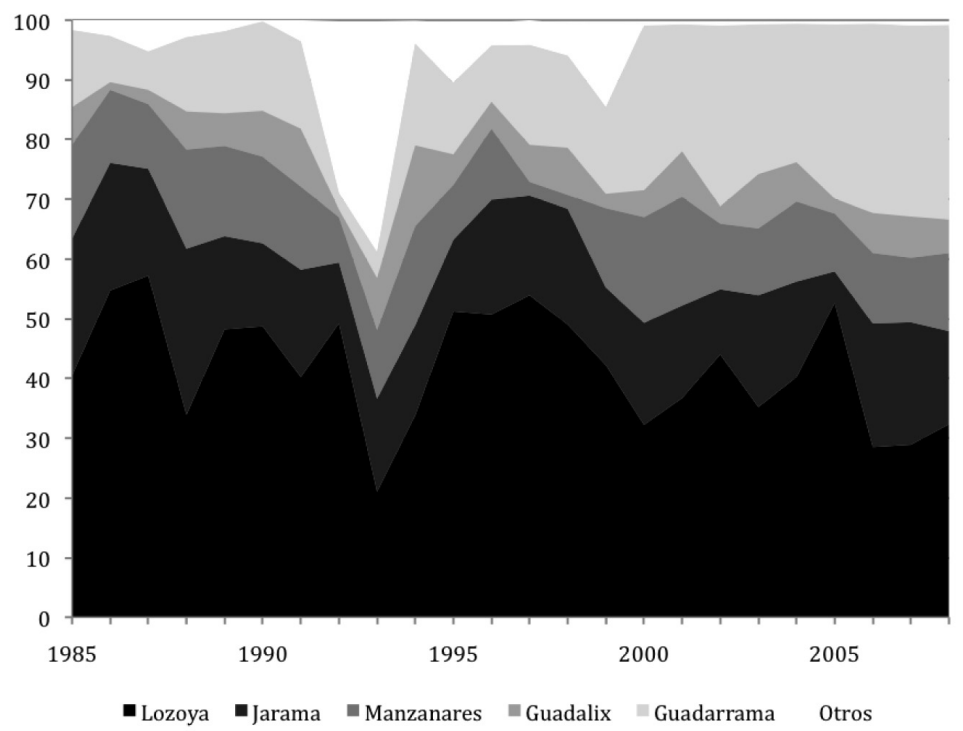

Figura 5. Origen del agua por cuencas consumida en la CAM, en porcentaje, 1985-2008. Fuente: elaboración propia a partir de datos del Instituto de Estadística de Madrid y del Canal de Isabel II.

\subsection{El Nuevo estructuralismo hidráulico: desalación}

Como ya se ha comentado anteriormente, la desalación constituyó la alternativa elegida por el PSOE para compensar los déficits hídricos que podía acarrear la cancelación del trasvase del Ebro previsto en el PHN de 2001. El denominado programa «AGUA» preveía la construcción de una treintena de desaladoras en el litoral mediterráneo y en las islas Baleares con una capacidad total más o menos equivalente a la del trasvase del Ebro. Las ventajas de la desalación resultaban indudables: acceder a los vastos reservorios del mar convertía este recurso en inagotable a todos los efectos con independencia del clima; la tecnología y particularmente el sistema de ósmosis inversa había reducido considerablemente los requerimientos energéticos y, por último y quizás más importante en el contexto español, se trataba de un recurso libre de controversias territoriales. En el haber de la desalación, por el contrario, deben apuntarse los costes energéticos (y el aumento de las emisiones de gases de efecto invernadero si se utiliza energía fósil) y el impacto ambiental de los residuos salinos en el medio marino. En términos económicos, la comparación entre la desalación y un trasvase como el previsto del Ebro no parece arrojar resultados concluyentes en uno u otro sentido. Sea como fuere, la desalación no puede considerarse como un cambio de paradigma respeto a la construcción de embalses y trasvases puesto que continúa siendo una opción destinada a aumentar la oferta de agua disponible.

En el caso de Barcelona, la desaladora de El Prat de Llobregat, finalizada en 2009 con un coste económico de 260 millones de euros, añade $60 \mathrm{hm}^{3}$ anuales a la oferta de agua para la Región Metropolitana. Además, se está ampliando la desaladora de Blanes (para suplir esencialmente a los núcleos turísticos y urbanos de la costa norte de la región) y se prevé construir otra desaladora en la desembocadura del Foix, en la costa sur (cerca 
de Tarragona). Cuando todos estos proyectos se encuentren finalizados, la oferta de agua para la RMB habrá aumentado en unos $160 \mathrm{hm}^{3}$ anuales. Además, la desaladora del Foix se conectará a la red del Consorcio de Aguas de Tarragona para suministrar agua a esta capital por si fallaran los caudales del Ebro. Según la ACA, estos nuevos recursos deben poder satisfacer la demanda de la RMB durante los próximos veinte años ahuyentando así el fantasma de posibles penurias hídricas futuras, incluidas las que pudieran derivarse del cambio climático.

Lejos de ser una alternativa relegada a las localidades costeras, la tecnología asociada a la desalación (principalmente la ósmosis inversa) se utiliza para la potabilización superficiales con alto contenido de sales minerales (en Barcelona dos plantas de potabilización en el Llobregat utilizan tecnología de desalación). De la misma manera se puede utilizar para potabilizar aguas subterráneas contaminadas o incluso en el proceso de reutilización de agua.

\subsection{Gestión de la demanda y recursos alternativos}

El modelo basado en un aumento de la oferta de agua mediante la construcción de grandes infraestructuras se ve crecientemente cuestionado por motivos económicos, sociales y ambientales. Además, la Directiva Marco del Agua de la Unión Europea (Comisión Europea 2000) exige la consideración de otras medidas de gestión, especialmente de tipo económico, para regular el consumo de este recurso. Todo ello ha puesto en evidencia la necesidad de avanzar hacia un enfoque integrado en la planificación y gestión de los recursos hídricos que incida sobre todo en el control de los usos del agua. Para el caso del agua urbana, las políticas de gestión de la demanda se pueden concretar en tres grandes ámbitos: el ámbito tecnológico, con instrumentos orientados a mejorar la eficiencia y el ahorro de agua en las redes de distribución y en hogares e industrias; el ámbito económico, con políticas tarifarias que incorporen todos los costes derivados del ciclo hidrológico urbano, y, finalmente, las acciones de concienciación ciudadana dirigida a mejorar los hábitos en el consumo de agua. Además, en estas políticas de gestión de la demanda cabría incluir la reutilización y también el aprovechamiento de recursos como las aguas pluviales. De los tres ámbitos es, sin duda, el económico donde más se ha progresado y el precio e impuestos del agua en Madrid y Barcelona se va acercando al principio de recuperación de costes que exige la Directiva Marco del Agua. En estos momentos y al menos para el caso de Barcelona, las tarifas ya son lo bastante elevadas como para incidir en un cierto descenso de los consumos más suntuarios como el riego de césped de jardín. Sin embargo, incrementos mayores como los que se barajan para cubrir el coste económico de la desalación pueden repercutir negativamente en las capas urbanas más desfavorecidas. En cuanto al ámbito tecnológico, durante los últimos años se viene produciendo una mejora sustancial en la eficiencia de las redes de abastecimiento a las ciudades, tal y como reflejan las encuestas del INE. Además debe tenerse en cuenta que las denominadas «pérdidas» pueden incluir agua no registrada por fallos o fraudes en los contadores, así como agua destinada a usos municipales. En cualquier caso, tanto en Madrid como en Barcelona, la eficiencia del sistema se sitúa por encima del $80 \%$. Por lo que se refiere a la penetración de equipamiento doméstico más eficiente en el uso de agua, cabe señalar también un peso creciente aunque difícil de cuantificar de grifería, inodoros, lavadoras y lavaplatos de bajo consumo. Sin embargo y para que esto último sea realmente significativo, debería pasar a formar parte de las regulaciones urbanísticas y de los códigos de construcción de viviendas promulgados por las corporaciones locales. Ejemplos como los de Leganés y Alcorcón, en Madrid, o como el de los 
municipios integrados en la denominada «Xarxa de Ciutats $i$ Pobles cap a la Sostenibilitat de la Diputación de Barcelona atestiguan la importancia de estas medidas que pueden extenderse también al uso de aguas pluviales o aguas recicladas para el riego de jardines y otros usos que no precisen de calidades óptimas. Finalmente, la concienciación ciudadana acerca de hábitos de consumo de agua más frugales resulta también una estrategia empleada profusamente en ambas ciudades y que, al menos en el caso de Barcelona y según la Entidad Metropolitana del Medio Ambiente de esta capital, ha permitido reducir los consumos por cápita de manera importante durante los últimos años.

\section{Modelos de gobernanza del agua urbana}

La gestión de la infraestructura descrita anteriormente sigue unos patrones bastante complejos que distinguen de manera notable ambas áreas. Es importante diferenciar entre la gestión y la titularidad ya que la legislación española (Ley estatal 7/1985, de 2 de abril, reguladora de las Bases de Régimen Local; Ley 30/2007, de 30 de octubre, de Contratos del Sector Público) permite distintos grados de participación privada en el ciclo del agua, que van desde la gestión comercial a contratos de concesión de larga duración (para una definición sistemática de los distintos tipos de contratos en la gestión del agua ver World Bank, 1997).

En esta parte del artículo, se presentará de manera resumida las distintas arquitecturas de gobierno del flujo de agua urbana. Por gobernanza entendemos la relación entre sociedad y su gobierno, en términos económicos, sociales y políticos. Paralelamente, Karen Bakker (2003) defiende el uso de la expresión «gobernanza» como el arte de conducir las sociedades. En este sentido, el concepto comprende el conjunto de procesos mediante los cuales los denominados stakeholders o agentes sociales articulan sus intereses, intentan imponer sus puntos de vista y controlan a los que toman las decisiones. Además, Furlong y Bakker (2007) distinguen el concepto de gobernanza al de gestión: mientras gobernanza se refiere al proceso de toma de decisiones, gestión se refiere a todo el operativo adoptado para alcanzar estas decisiones. Pueden existir distintos modelos de gobernanza de servicios esenciales como el agua urbana. Según ejemplos proporcionados por Bakker (2003) o McGranahan (2001) podemos encontrar modelos que giran alrededor de la planificación del Estado, modelos basados en el mercado o incluso modelos de gobernanza comunitaria (ver tabla 4).

En este artículo argumentamos que estos modelos presentan tendencias hacia la «hibridación»y pueden generar manifestaciones contradictorias. A grandes rasgos, podríamos decir que a principios de 2010 nos encontramos en una situación dicotómica por lo que representa el suministro urbano del agua en Barcelona y Madrid: mientras en el caso de Madrid, la gestión y titularidad es totalmente pública ${ }^{3}$ (desde la creación del suministrador moderno, el Canal de Isabel II) el caso de Barcelona se caracteriza por una importante participación privada en la obtención y distribución de agua potable (que se remonta a 140 años atrás). Sin embargo queremos subrayar que estos modelos presentan tendencias convergentes, sobretodo alrededor del modelo organizacional, como intentaremos desarrollar a continuación.

3 En el momento de redacción del artículo el Canal de Isabel II era de titularidad de la Comunidad de Madrid. Sin embargo desde Septiembre de 2008 se habla de un proceso de privatización parcial de la compañía pública. 
Tabla 4

MODELOS DE GOBERNANZA PARA LA PROVISIÓN DE SERVICIOS PÚBLICOS

\begin{tabular}{|c|c|c|c|}
\hline & Planificación & Mercado & Comunidad \\
\hline Titular infraestructura & Sector Público & Empresa privada & Usuarios \\
\hline Gestor infraestructura & Sector Público & Empresa privada & Usuarios \\
\hline Rol del consumidor & Ciudadanos & Clientes & $\begin{array}{l}\text { Miembros de la } \\
\text { comunidad }\end{array}$ \\
\hline Estructura organizativa & Funcionarial & $\begin{array}{l}\text { Empresa/ } \\
\text { Corporación }\end{array}$ & Asociación/Red \\
\hline Accountability $^{4}$ & Jerarquía & Contrato & $\begin{array}{l}\text { Normas } \\
\text { comunitarias }\end{array}$ \\
\hline $\begin{array}{l}\text { Instancias normativas } \\
\text { (decision makers) }\end{array}$ & $\begin{array}{l}\text { Administración, } \\
\text { Expertos, } \\
\text { Funcionarios }\end{array}$ & $\begin{array}{l}\text { Expertos, } \\
\text { Compañías, } \\
\text { Individuos }\end{array}$ & $\begin{array}{l}\text { Líderes y } \\
\text { miembros de las } \\
\text { organizaciones } \\
\text { comunitarias } \\
\end{array}$ \\
\hline Objetivo principal & $\begin{array}{l}\text { Minimizar el riesgo } \\
\text { Cumplir con los } \\
\text { requisitos legales y } \\
\text { políticos }\end{array}$ & $\begin{array}{l}\text { Maximizar los } \\
\text { ingresos } \\
\text { Actuación eficiente }\end{array}$ & \begin{tabular}{|l|} 
Servir a los \\
intereses de la \\
comunidad \\
Actuación \\
efectiva \\
\end{tabular} \\
\hline $\begin{array}{l}\text { Incentivos principales } \\
\text { para una buena gestión }\end{array}$ & $\begin{array}{l}\text { Opinión del } \\
\text { ciudadano } \\
\text { Feedback de los } \\
\text { expertos }\end{array}$ & $\begin{array}{l}\text { Señales de precios, } \\
\text { Opinión del } \\
\text { consumidor }\end{array}$ & $\begin{array}{l}\text { Comunidad } \\
\text { Normas } \\
\text { comunitarias }\end{array}$ \\
\hline $\begin{array}{l}\text { Amenazas principales } \\
\text { por no mantener el } \\
\text { servicio }\end{array}$ & $\begin{array}{l}\text { Proceso político a } \\
\text { través de elecciones } \\
\text { Litigio } \\
\text { Coerción del Estado }\end{array}$ & $\begin{array}{l}\text { Pérdida financiera } \\
\text { Absorción } \\
\text { Litigio }\end{array}$ & $\begin{array}{l}\text { Presión social } \\
\text { Litigio }\end{array}$ \\
\hline
\end{tabular}

Fuente: Adaptado de Bakker (2003) y McGranahan (2001).

\subsection{Lo público y lo privado en la gestión del agua en Barcelona}

En Barcelona la esfera pública y el capital privado se entremezclan para dar forma a una compleja estructura multiescalar de gobernanza del agua, que abarca desde el nivel local hasta el internacional, con distintos roles y con una marcada diferencia entre el regulador y el suministrador. Aún así, excluyendo las instancias regulatorias europeas (Directiva Marco del Agua) y estatales (Ley de Aguas y Ley de Régimen de Bases Local) que son comunes a ambos, para sistematizar podemos decir que cuando un ciudadano de Barcelona abre el grifo cuatro agentes sociales intervienen en este proceso: la Agència Catalana del Agua, la Entidad Metropolitana de Medi Ambient (EMMA)/Ayuntamientos y la Corporación Agbar.

4 Responsabilidad de rendir cuentas. 


\subsubsection{Agència Catalana de 1'Aigua}

La administración hidráulica en Catalunya está encabezada por la Agència Catalana de l'Aigua (ACA), una empresa pública de la Generalitat de Catalunya, adscrita al Departament de Medi Ambient i Habitatge. Tiene competencias plenas en las Cuencas Internas de Catalunya y competencias compartidas en las otras cuencas intercomunitarias. La ACA nació a finales de la década de los 1990, entre otros motivos, para superar el grave conflicto de la denominada Guerra de l'Aigua, que había supuesto que más de 60.000 familias del Área Metropolitana de Barcelona dejaran de pagar los tributos asociados a este recurso (ver Tello, 2000).

Entre las funciones de la ACA destaca la planificación del ciclo integral del agua basada en los principios de la Directiva Europea Marco de 2000. Aparte de los atributos típicos de un organismo de cuenca (concesiones, aprovechamientos hidráulicos, saneamiento, vigilancia, inspección, determinación de zonas inundables etc.), la Agencia grava con un impuesto («Cànon de l'Aigua») los usos domésticos e industriales para cubrir (parcialmente) los costes de saneamiento.

Una de las obligaciones de la ACA como organismo de cuenca es el abastecimiento en alta de la demanda urbana (tratamiento, almacenamiento y transporte de agua hasta las zonas a abastecer). Esta función es desarrollada en la RMB principalmente por otra empresa pública de la Generalitat de Catalunya: Aigües Ter-Llobregat (ATLL). ATLL se encarga de la planificación, construcción, gestión y explotación de la infraestructura del sistema TerLlobregat (ver tabla 2). En este sentido, ATLL dispone de dos estaciones de tratamiento de agua (ETAPs), una en el Llogregat y otras en el punto de distribución del agua procedente del trasvase del Ter. Aparte, se ha encargado de la construcción de la planta de desalinización de El Prat de Llobregat, aunque en la práctica ésta ha sido construida y gestionada por la compañía Agbar, de la que hablaremos posteriormente. Antes, sin embargo, no podemos obviar el importante papel de los municipios y mancomunidades en el suministro de agua.

\subsubsection{Municipios/Entidad Metropolitana de Medi Ambient (EMMA)}

La distribución de agua a los domicilios (en baja) es una competencia municipal según la legislación española. Sin embargo los municipios pueden mancomunarse para desarrollar esta función. A su vez, los municipios/mancomunidades pueden cumplir esta función de manera indirecta.

Los municipios integrantes del Área Metropolitana de Barcelona (AMB) tienen delegadas sus funciones de suministro hídrico (abastecimiento en baja y saneamiento) a la Entitat del Medi Ambient (también llamada Entitat Metropolitana de Serveis Hidráulics i Tractament de Residus). A su vez esta entidad tiene delegado el suministro a la Sociedad General de Aguas de Barcelona, integrada en el grupo Agbar.

\subsubsection{Sociedad General de Aguas de Barcelona (Grupo Agbar)}

Desde hace más de 140 años la Sociedad General de Aguas de Barcelona ha suministrado agua a la ciudad de Barcelona. Con el paso de los años ha ido ampliando su ámbito geográfico hasta convertirse en una de las mayores empresas privadas del sector a nivel mundial. Como hemos dicho, en el AMB abastece la mayor parte de los municipios y a través de sus filiales también a la mayor parte del resto de los municipios de la RMB.

Si bien su rol principal es el de suministrador en baja, Agbar también participa en el abastecimiento en alta ya que dispone de una planta potabilizadora en el Llobregat así como 
varios pozos en esta cuenca y en la del Besòs. Desde el verano de 2009 explota la planta desalinizadora del Llobregat fruto de un contrato con ATLL de construcción, explotación y transferencia (BOT en inglés).

Hasta 2009 la mayor parte de la compañía estaba controlada por La Caixa, seguida por Suez Environnement. En 2010 se materializó la venta de parte de las acciones de La Caixa a Suez, que se ha convertido en el propietario mayoritario de Agbar, lo que significa un cambio importante en lo que se refiere al poder decisional trasladado desde Barcelona a París (aunque la sede de Agbar permanece en Barcelona).

\subsection{Canal de Isabel II: controlando el ciclo integral del agua en Madrid}

En Madrid, las diferencias entre el regulador, el suministrador en alta y el suministrador en baja son más difusas. Existe un nombre propio alrededor del cual gira toda la gestión integral del ciclo del agua: el Canal de Isabel II.

Como ya hemos comentado anteriormente, el Canal de Isabel II se creó a mitad del siglo XIX para dar respuesta a los graves problemas hídricos que padecía la capital. Su carácter público ha prevalecido durante más de 150 años, pero su estructura de gobierno ha ido cambiando con el paso del tiempo. Desde 1984 es una empresa pública perteneciente a la Comunidad Autónoma de Madrid (CAM) y abastece (en alta y en baja) virtualmente a todos los municipios de la región. También se encarga del saneamiento y asume ciertas funciones de mejora ambiental.

El Canal de Isabel II, aún y ser una empresa pública, ha seguido una expansión similar a la de la Agbar, y desde la década de los 1990 ha penetrado en América Latina y más recientemente también ha empezado a realizar operaciones comerciales en China.

Por su parte, también tenemos que mencionar la administración hidráulica competente, la Confederación Hidrográfica del Tajo, organismo de cuenca que tiene entre sus funciones la vigilancia, sanción, concesión o planificación (similares a los anteriormente mencionados para la Agència Catalana de l'Aigua). Aún así recordamos la particularidad de la situación, ya que el Canal de Isabel II, en tanto que empresa pública, tiene competencias de planificación y abastecimiento y tiene una concesión gratuita para abastecer a los ciudadanos de la CAM.

En septiembre de 2008 desde el Gobierno de la CAM se anunció la idea de cambiar la configuración legal del Canal de Isabel II, permitiendo la entrada de capital privado. La lógica de realizar este cambio se fundamentaba en una teórica necesidad de ampliación de capital para afrontar nuevos retos de abastecimiento y de preservación ambiental. Este proceso tendría dos fases distintas, una primera de transformación del Canal en una Sociedad Anónima y la posterior salida a bolsa de parte de las acciones de la empresa. Ello es objeto de un enconado debate en Madrid sin que hasta el momento se haya materializado ninguna propuesta concluyente en relación a la privatización.

\section{Conclusiones y perspectivas de futuro}

En este artículo se ha intentado aportar de manera sintética la génesis y evolución de la urbanización del suministro hídrico moderno en las dos áreas urbanas más importantes del estado, Madrid y Barcelona. A su vez se han presentado las estructuras actuales de gobernanza así como los cambios más destacados acaecidos recientemente.

En Barcelona, la reciente modificación en la estructura accionarial de Agbar, combinado con el creciente déficit al que se enfrenta el regulador (ACA) abre la posibilidad a nuevos escenarios y a una reconfiguración del ciclo hidrosocial, que se suma también al nuevo escenario abierto por la desalinización. 
Madrid puede seguir unos pasos similares y de ahí que podríamos hablar de una tendencia convergente en la gobernanza del ciclo hidrológico en ambas áreas. Desde el traspaso del Canal de Isabel II a la CAM en la década de 1980, la empresa ha seguido un proceso de internacionalización y corporatización que la sitúan en una posición intermedia en los modelos de gobernanza que hemos explicitado anteriormente (tabla 4). A su vez la entrada de capital privado en la compañía madrileña podría aún aproximar más el modelo de gobernanza del agua de Madrid al de Barcelona.

Sin embargo este proceso de privatización parcial o entrada de capital privado traería un cambio de naturaleza legal del suministrador. Este proceso tendría que ser aprobado por la administración central a través de la Confederación Hidrográfica del Tajo (CHT) (ver El País. es, 9/10/08, «Un informe estatal considera casi inviable la privatización del Canal») ya que parte de la infraestructura (así como la concesión de aprovechamiento hídrico) pertenece al dominio público. De la misma manera los ayuntamientos tendrían que renegociar las condiciones de concesión del suministro hídrico, como ya anunció la misma ciudad de Madrid (ver por ejemplo artículo 16, Convenio de relaciones entre el Ayuntamiento de Madrid y el Canal de Isabel II en materia de abastecimiento de agua para la ciudad de Madrid, 2005).

Concluimos argumentando que ambos procesos se pueden enmarcar en una tendencia global hacia la neoliberalización y privatización de los recursos naturales y del medio ambiente debido a la creciente necesidad de encontrar nuevas esferas para la acumulación y circulación de capital (ver por ejemplo Castree 2003, 2006, 2008a, 2008b, Heynen et al., 2007, Heynen y Robbins, 2005). El agua no escapa a esta lógica, y el flujo de capital se imbrica con el flujo hídrico para configurar nuevas realidades socionaturales. Se tendrá que esperar, sin embargo, a ver cuales son los resultados finales de estos procesos ya que es pronto para aventurarse a hacer hipótesis. Sin embargo, coincidiendo con Peck y Tickell (2007) queremos resaltar la importancia de la escala local y regional en la configuración de las nuevas realidades políticas y económicas; Madrid y Barcelona configuran un «nuevo localismo» (Brenner y Theodore, 2002a, 2002b) que puede determinar de manera crítica los resultados de ambos procesos. Entender el funcionamiento y los cambios de configuración en la gobernanza de este recurso va mucho más allá de un mero interés científico o académico. Una comprensión adecuada de este sistema puede contribuir a dibujar futuros urbanos socialmente más justos y ambientalmente más sostenibles.

\section{Bibliografía}

ALGABA, A., 2001. Las nuevas dinámicas metropolitanas. el estudio de los cambios acontecidos en el Área Metropolitana de Barcelona a través de algunas publicaciones recientes. Revista Bibliográfica De Geografía y Ciencias Sociales, 271.

ALLAN, J.A., 1998. Virtual Water: A Strategic Resource. Global Solutions to Regional Deficits. Groundwater, 36:545-546.

ARIAS, A. y BORJA, J., 2005. Metropolitan cities: Territory and governability, the Spanish case. Built Environment, 33(2), 170-184.

ARROJO, P., 2003. El Plan Hidrológico Nacional: una cita frustrada con la historia. RBA, Barcelona, $207 \mathrm{pp}$.

BAKKER, K. (Editor), 2003. Good governance in restructuring water supply: A handbook. Federation of Canadian Municipalities and Program on Water Issues, Ottawa, $44 \mathrm{pp}$.

BRENNER, N. y THEODORE, N., 2002a. Cities and the geographies of 'actually existing neoliberalism'. Antipode, 34:349-379.

BRENNER, N. y THEODORE, N., 2002b. Preface: from the 'new localism' to the spaces of Neoliberalism. Antipode, 34:341-347.

CAPEL, H., 2007. El debate sobre la construcción de la ciudad y el llamado» Modelo Barcelona. Scripta Nova, 233:1 de noviembre de 2009, Disponible en: http://www.ub.es/geocrit/sn/sn-233.htm 
CASTREE, N., 2003. Commodifying what nature? Progress in Human Geography, 27:273.

CASTREE, N., 2006. From neoliberalism to neoliberalisation: consolations, confusions, and necessary illusions. Environment and Planning A, 38:1-6.

CASTREE, N., 2008a. Neoliberalising nature: the logics of deregulation and reregulation. Environment and Planning A, 40:131-152.

CASTREE, N., 2008b. Neoliberalising nature: processes, effects, and evaluations. Environment and Planning A, 40:153-173.

COMISIÓN EUROPEA, 2000. EU Water Framework Directive. Directive 2000/60/EC.

CHAPAGAIN, A.K., HOEKSTRA, A.Y. y SAVENIJE, H.H.G., 2005. Saving water through global trade. Value of Water Research Report Series No.17, IHE-UNESCO, Delft, 36 pp.

DOMENE, E., SAURÍ, D. y PARÉS, M., 2005. Urbanization and sustainable resource use: The case of garden watering in the metropolitan region of Barcelona. Urban Geography, 26:520-535.

DOMENE, E. y SAURÍ, D., 2006. Urbanisation and water consumption: Influencing factors in the Metropolitan Region of Barcelona. Urban Studies, 43:1605-1623.

DOMENE, E. y SAURÍ, D., 2007. Urbanization and class-produced natures: Vegetable gardens in the Barcelona Metropolitan Region. Geoforum, 38:287-298.

FACTORS IN THE METROPOLITAN REGION OF BARCELONA. Urban Studies, 43:1605-1623.

EUROPEAN ENVIRONMENT AGENCY, (EEA), 2005. European Environmental Outlook. Report 4/2005. European Environment Agency, Copenhagen, 87 pp.

EUROPEAN ENVIRONMENT AGENCY, (EEA), 2006. Urban Sprawl in Europe. The ignored challenge. Report 10/2006. European Environment Agency, Copenhagen, 56 pp.

FURLONG, K. y BAKKER, K., 2007. Water Governance in Transition: Utility restructuring and water efficiency in Ontario. Policy Report, September 2007, University of British Columbia Program on Water Governance, $30 \mathrm{pp}$.

GANDY, M., 2002. Concrete and clay: reworking nature in New York City. MIT Press, Cambridge, Massachusetts; London, $344 \mathrm{pp}$.

GARCÍA PALOMARES, J.C. y GUTIÉRREZ PUEBLA, J., 2007. La ciudad dispersa: cambios recientes en los espacios residenciales de la Comunidad de Madrid. Anales de Geografía, 27:45-67.

GIL OLCINA, A. y RICO AMORÓS, A.M., 2008. Políticas del Agua III. De la Ley de Aguas de 1985 al PHN. Murcia, ESAMUR.

HEYNEN, N., McCARTHY, J., PRUDHAM, S. y ROBBINS, P. (Editores), 2007b. Neoliberal Environments: False promises and unnatural consequences. Routlegde, London; New York, $298 \mathrm{pp}$.

HEYNEN, N. y ROBBINS, P., 2005. The Neoliberalization of Nature: Governance, Privatization, Enclosure and Valuation. Capitalism Nature Socialism, 1:5-8.

HOEKSTRA, A.Y. y CHAPAGAIN, A.K., 2007. Water footprints of nations: water use by people as a function of their consumption pattern. Water Resources Management, 21:35-48.

KALLIS, G., 2008. Droughts. Annual Review of Environment \& Resources, 33:85-118.

LÓPEZ DE LUCIO, R., 2003. Transformaciones territoriales recientes en la región urbana de Madrid. Urban, 8:124-161.

MARCH, H., PERARNAU, J. y SAURÍ (en prensa). Exploring the links between immigration, ageing, and domestic water consumption: the case of the Metropolitan Area of Barcelona, Regional Studies.

MARCH, H, and SAURÍ, D., 2010. The Suburbanization of Water Scarcity in the Barcelona Metropolitan Region: Sociodemographic and Urban Changes Influencing Domestic Water Consumption. The Professional Geographer, 62:32-45.

MARCH, H. y SAURÍ, D. 2009. What lies behind domestic water use? A review essay on the drivers of domestic water consumption. Boletín de la Asociación Española de Geógrafos Españoles, 50:297-314.

MARTÍNEZ VÁZQUEZ DE PARGA, Rosario, 2001a. Historia del Canal de Isabel II. Ediciones del Aniversario. Fundación Canal de Isabel II, Madrid, 357 pp.

McGRANAHAN, G., 2001. The citizens at risk: from urban sanitation to sustainable cities. Earthscan, London; Sterling, 200 pp.

NAREDO, J.M. (coord.), CARPINTERO, O., FRÍAS, J., GASCÓ, J.M. y SAA, A., 2008. El agua virtual y la huella hidrológica en la Comunidad de Madrid. Cuadernos I+D+I ${ }^{\circ} 5$, Canal de Isabel II, Madrid, 183 pp. 
NAREDO, J.M. y GARCÍA ZALDÍVAR, R., 2008. Estudio sobre la ocupación de suelo por usos urbano-industriales, aplicado a la Comunidad de Madrid. Informe final. Convenio de Colaboración entre la Universidad Politécnica de Madrid y el Ministerio de Medio Ambiente y Medio Rural y Marino, Madrid, 103 pp.

PARÉS-FRANZI, M., SAURÍ, D. y DOMENE, E., 2006. Evaluating the Environmental Performance of Urban Parks in Mediterranean Cities: An Example from the Barcelona Metropolitan Region. Environmental Management, 38:750-759.

PECK, J. y TICKELL, A., 2007. Conceptualizing Neoliberalism, Thinking Tatcherism. In: H. Leitner, J. Peck and E.S. Sheppard (Editors), Contesting Neoliberalism: urban frontiers. The Guilford Press, New York, pp. 26-50.

PLATA ROCHA, W., GÓMEZ DELGADO, M. y BOSQUE SENDRA, J., 2009. Cambios de usos del suelo y expansión urbana en la Comunidad de Madrid (1990-2000). Scripta Nova, 13:15 de junio de 2009. Disponible en: http://www.ub.es/geocrit/sn/sn-293.htm

SÁNCHEZ, A., 2003. Aproximació a l'estudi de les migracions residencials a la regió metropolitana de barcelona. els casos d'Alella i Matadepera. Revista Catalana De Sociologia, 19, 161-188.

TELLO, E., 2000. 'La guerra del agua' en Barcelona. Alternativas económico-ecológicas para un desafio socioambiental. En: A. Estevan and V. Viñuales (Editors), La eficiencia del agua en las ciudades. Bakeaz Fundacion Ecología y Desarrollo, Bilbao, pp. 277-298.

WORLD BANK, 1997. Toolkits for private participation in water and sanitation. World Bank, Washington, D.C., 43 pp. 